\title{
Tunable Wigner States with Dipolar Atoms and Molecules
}

\author{
J. C. Cremon, ${ }^{1}$ G. M. Bruun,,${ }^{1,2}$ and S. M. Reimann ${ }^{1}$ \\ ${ }^{1}$ Mathematical Physics, LTH, Lund University, SE-22100 Lund, Sweden \\ ${ }^{2}$ Department of Physics and Astronomy, University of Aarhus, DK-8000 Aarhus C, Denmark
}

\begin{abstract}
We study the few-body physics of trapped atoms or molecules with electric or magnetic dipole moments aligned by an external field. Using exact numerical diagonalization appropriate for the strongly correlated regime, as well as a classical analysis, we show how Wigner localization emerges with increasing coupling strength. The Wigner states exhibit nontrivial geometries due to the anisotropy of the interaction. This leads to transitions between different Wigner states as the tilt angle of the dipoles with the confining plane is changed. Intriguingly, while the individual Wigner states are well described by a classical analysis, the transitions between different Wigner states are strongly affected by quantum statistics. This can be understood by considering the interplay between quantum-mechanical and spatial symmetry properties. Finally, we demonstrate that our results are relevant to experimentally realistic systems.
\end{abstract}

Recent experimental advances in cold quantum gases have placed focus on atoms or molecules with permanent dipole moments. A Bose-Einstein condensate of ${ }^{52} \mathrm{Cr}$ atoms has been realized [1, 2, and recently Dy atoms were cooled and trapped [3]. These atom species have magnetic dipole moments of several Bohr magnetons. A promising development is the trapping and cooling of diatomic molecules with electric dipole moments [4] 6 . The realization of a molecular fermionic ${ }^{40} \mathrm{~K}^{87} \mathrm{Rb}$ gas was a significant breakthrough [7. Dipolar gases offer access to a broad range of novel few- and many-body physics, in single traps as well as in optical lattices which has spurred intensive theoretical interest (see the recent reviews by Baranov [8] and Lahaye et al. 9]). The attractive part of the dipolar interaction leads to a collapse instability in three dimensions [9]. A remedy is to use traps of reduced dimensionality. For instance, the interaction between dipoles in a $2 \mathrm{D}$ plane is predominantly repulsive when they form a sufficiently large angle with respect to the plane (see Fig. 1). This stabilizes the system against collapse [10. A variety of interesting many-body states for dipoles in 2D has been examined theoretically [10 12 .

Here, we examine a 2D system of this kind in the regime of strong repulsive interactions using exact diagonalization as well as a classical analysis. Contrary to the analogous Wigner states of electrons in metals 14

and quantum dots [15] the anisotropic dipolar interaction is shown to give rise to Wigner states with nontrivial geometries that depend on the alignment angle. This leads to transitions between different geometries that are crucially influenced by quantum statistics. We finally argue that our results are experimentally observable.

We consider particles with mass $m$ and a magnetic (electric) dipole moment $\mu(d)$ which is aligned by an external field such that it lies in the $x z$ plane, forming an angle $\Theta$ with the $x$ axis (see Fig. 1). The interaction between two electric dipoles separated by a vector $\mathbf{r}$ is

$$
V(\mathbf{r})=\frac{d^{2}}{4 \pi \epsilon_{0}} \frac{1-3 \cos ^{2} \theta_{r d}}{r^{3}}
$$

where $\theta_{r d}$ is the angle between a dipole moment and $\mathbf{r}$. The particles are confined in the $x y$-plane by a quasi-two-
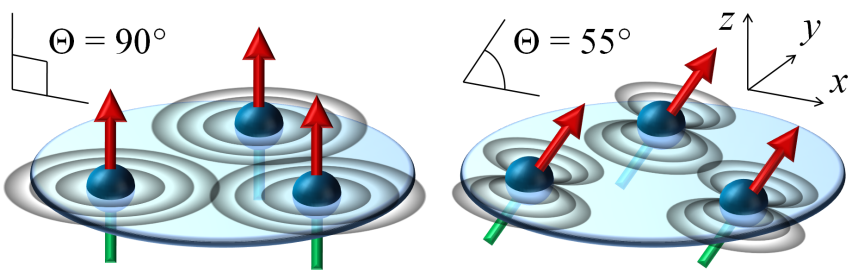

Figure 1: Dipolar particles in a quasi-2D trap in the $x y$ plane. The gray circles indicate the contours of the effective interaction potentials in the plane. In the left-hand panel the dipoles are perpendicular $\left(\Theta=90^{\circ}\right)$ to the plane of motion and the interaction is isotropic in the plane, while in the right-hand panel the dipoles are tilted $\left(\Theta=55^{\circ}\right)$ and the interaction is anisotropic.

dimensional harmonic trap $V_{\text {trap }}(x, y, z)=m\left[\omega_{0}^{2}\left(x^{2}+\right.\right.$ $\left.\left.y^{2}\right)+\omega_{z}^{2} z^{2}\right] / 2$, with $\omega_{z} \gg \omega_{0}$ so that the particles are in the ground state orbital of the $z$ direction. The corresponding oscillator lengths are $l_{z}$ and $l_{0}$ with $l_{z} \ll l_{0}$. Throughout this Letter, we consider dipoles with no internal spin degrees of freedom.

The effective interaction $V_{2 D}(r, \phi)$ in the $x y$-plane is obtained by integrating out the harmonic motion in the $z$-direction. This yields

$$
\begin{aligned}
& \frac{V_{2 \mathrm{D}}(r, \phi)}{\hbar \omega_{0}}=\frac{D^{2}}{2 \sqrt{2 \pi}} \frac{e^{\xi / 2}}{\left(l_{z} / l_{0}\right)^{3}}\left((2+2 \xi) K_{0}(\xi / 2)-2 \xi K_{1}(\xi / 2)\right. \\
& +\cos ^{2} \Theta\left[-(3+2 \xi) K_{0}(\xi / 2)+(1+2 \xi) K_{1}(\xi / 2)\right]+ \\
& \left.+2 \cos ^{2} \Theta \cos ^{2} \phi\left[-\xi K_{0}(\xi / 2)+(\xi-1) K_{1}(\xi / 2)\right]\right)
\end{aligned}
$$

where $\xi=r^{2} /\left(2 \tilde{l}_{z}^{2}\right)$ and $K_{0}$ and $K_{1}$ are irregular modified Bessel functions. (For the special cases $\Theta=90^{\circ}$ and $\Theta=$ $0^{\circ}$, expressions are also given in [12, 16].) For electric dipoles, $D=\frac{d}{\sqrt{4 \pi \epsilon_{0}}} \frac{\sqrt{m}}{\hbar \sqrt{l_{0}}}$ whereas $D=\mu \sqrt{\frac{\mu_{0}}{4 \pi}} \frac{\sqrt{m}}{\hbar \sqrt{l_{0}}}$ for magnetic dipoles. The effective interaction (2) reduces to the bare interaction (1) when $r \gg l_{z}$. Depending on the angle $\Theta, V_{2 \mathrm{D}}(r, \phi)$ contains both attractive and repulsive regions. For $\Theta=90^{\circ}$ it is isotropic in the $x y$ - 
plane, whereas it is anisotropic with increasing attractive regions for decreasing angle. Since the focus is on Wigner states in this Letter, we restrict our analysis to the case $\arccos \frac{1}{\sqrt{3}} \approx 54.7^{\circ}<\Theta \leq 90^{\circ}$, for which the interaction without the $z$-integration is purely repulsive.

Our numerical results are obtained by exact diagonalization using a basis of 2D harmonic oscillator orbitals. The multiparticle basis space is truncated by including all states with (kinetic and potential) energy up to some cutoff value $E_{\text {cut }}$. Here, good convergence is achieved at $E_{c u t}<20 \hbar \omega$, and variations of $E_{c u t}$ do not qualitatively affect our conclusions. Because of the computational effort involved, the method is limited to particle numbers $N \lesssim 4$. In the following we set $l_{z} / l_{0}=0.1$. Our results are not sensitive to this ratio as long as it is sufficiently small. In Fig. 2 we plot the particle density of the ground state for two different tilt angles $\Theta$ for a system with $N=3$ dipolar bosons and fermions. For weak coupling, $D=0.1$, the dipoles essentially form an ideal gas. The dip in the density for the fermions in Fig. 2 is simply a shell effect. With increasing interaction, the dipoles localize in Wigner states. Comparing the interaction energy with the confinement kinetic energy yields the condition $D^{2} \gg 1$ for Wigner crystallization. Our numerical results confirm this showing that the crystallized structure emerges continuously for $D \gtrsim 1$ both for bosons and fermions for this finite-size system; for $D=5$ the localization can be clearly seen in Fig. 2.

The Hamiltonian and the density of the ground state have rotational symmetry for $\Theta=90^{\circ}$. To demonstrate particle localization, we therefore study the paircorrelated density $\rho\left(\mathbf{r}, \mathbf{r}^{\prime}\right)=\left\langle\hat{\psi}^{\dagger}(\mathbf{r}) \hat{\psi}^{\dagger}\left(\mathbf{r}^{\prime}\right) \hat{\psi}\left(\mathbf{r}^{\prime}\right) \hat{\psi}(\mathbf{r})\right\rangle$. Fixing one particle at position $\mathbf{r}$, it gives the probability distribution of the remaining $N-1$ particles. We see from Fig. 2 that for strong interactions and $\Theta=90^{\circ}$, the particles localize at the vertices of an equilateral triangle. For $\Theta=55^{\circ}$, the interaction is anisotropic in the $x y$-plane and almost vanishing between two dipoles whose relative vector is parallel to the $x$-axis. Figure 2 shows that the ground state for $D=5$ features the three particles on a straight line for both bosons and fermions. This exploits the weaker regions of the interaction and minimizes the energy. A classical analysis minimizing the trap and interaction energies of three dipoles in a 2D trap indeed yields that the lowest energy configuration is all particles on a line along the $x$-axis for $\Theta \lesssim 62^{\circ}$ and the three particles in an equilateral triangle for $\Theta \gtrsim 62^{\circ}$.

We now examine the transition from a line shaped to a triangular shaped ground state with increasing angle. Figure 3 depicts the lowest energy states of the three dipoles and their density as a function of the tilt angle $\Theta$. In agreement with the classical analysis, the ground state changes from a linear to a triangular Wigner state as $\Theta$ is increased. For $D=5$, the transition occurs at $\Theta \simeq 56^{\circ}$ for fermions and $\Theta \simeq 57^{\circ}$ for bosons which is somewhat lower than the classical prediction $\Theta \simeq 62^{\circ}$ due to quantum fluctuations. The density of the triangular state appears rotationally symmetric since the energy of

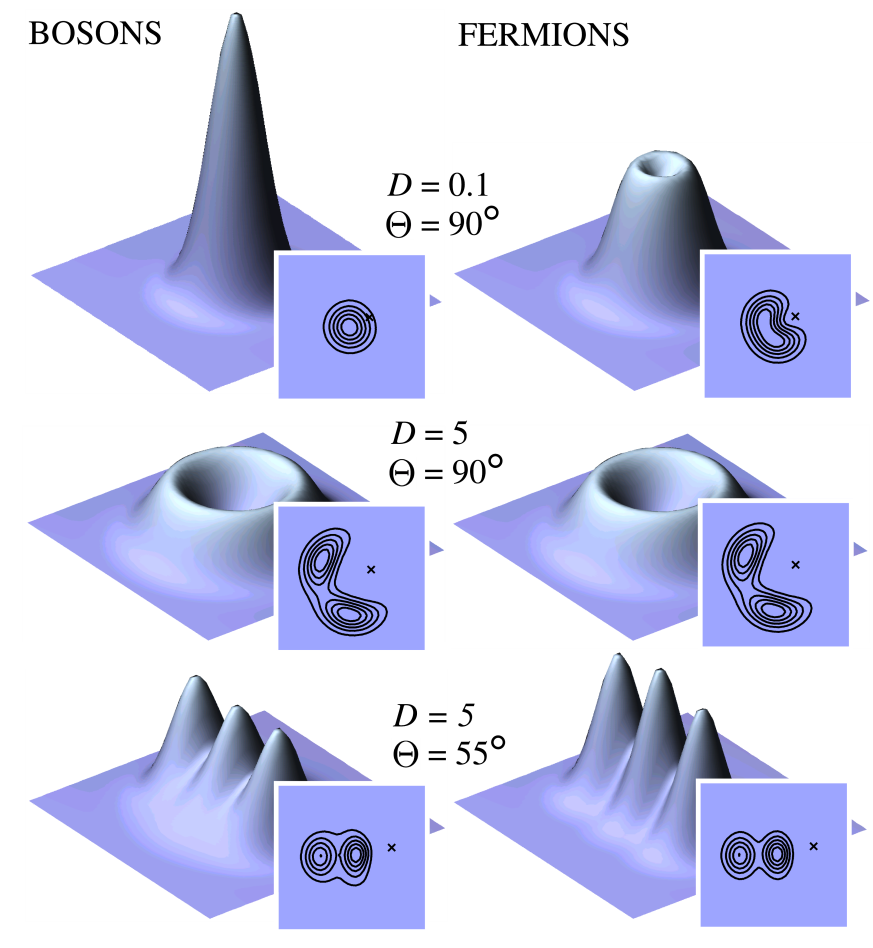

Figure 2: Particle densities for three different coupling strengths, with dipole tilt angles $\Theta=90^{\circ}$ and $\Theta=55^{\circ}$. The insets show the pair-correlated density functions; the crosses mark the position of one particle. The $x$ and $y$ axes range from $-4 l_{0}$ to $4 l_{0}$, both here and in Figs. 3 and 4 .

three aligned classical dipoles in an equilateral triangle can be shown to be independent of its orientation.

There is an intriguing difference between the transitions from linear- to triangular-shaped Wigner states for fermionic and bosonic dipoles: For fermions, the transition is a sharp crossing, whereas, for bosons, it is a continuous mixing of the triangular and linear states corresponding to an avoided crossing. This can be understood by considering that the Hamiltonian is invariant under the mirror operations $\hat{P}_{x}:(x, y) \rightarrow(-x, y)$ and $\hat{P}_{y}:$ $(x, y) \rightarrow(x,-y)$. The energy eigenstates must therefore also be eigenstates of $\hat{P}_{x}$ and $\hat{P}_{y}$ with eigenvalues \pm 1 . The key point is that for the Wigner states, one can infer their eigenvalues under these symmetry operations from their quantum statistics. Consider the threeparticle line configuration discussed above. Applying $\hat{P}_{x}$ simply corresponds to exchanging the coordinates of the two outer localized particles. For bosons, this must give a plus sign due to the symmetrization of the wave function. For fermions one must correspondingly get a minus sign. Likewise, since all three particles have a finite probability to appear on the $x$-axis, the eigenvalue for $\hat{P}_{y}$ must be +1 for both bosons and fermions. We write this as $\left(P_{x}, P_{y}\right)_{\mathrm{B}, \text { line }}=(+,+)$ for the bosonic line state and $\left(P_{x}, P_{y}\right)_{\mathrm{F}, \text { line }}=(-,+)$ for the fermionic line state.

The triangular Wigner state is more subtle, as it contains components with the triangle in all possible orien- 

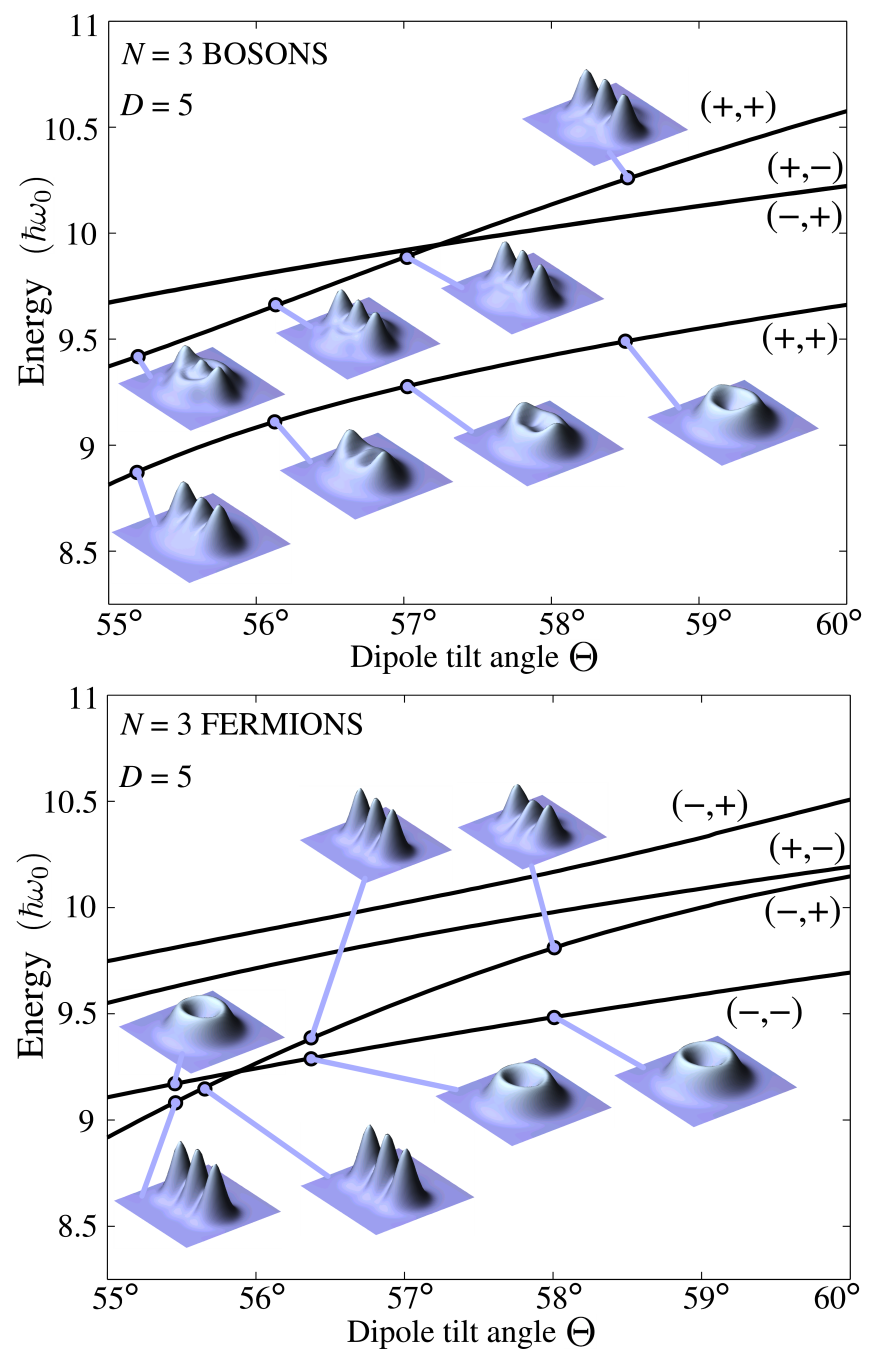

Figure 3: The four lowest energy states for three strongly interacting bosonic (top panel) and fermionic (bottom panel) dipoles. By changing the dipole tilt angle $\Theta$, the ground state changes from a Wigner state with line geometry to one with triangular geometry. The signs in brackets denote $x$ - and $y$ parities, as explained in the text.

tations. Take the component with one of the vertices of the triangle lying on the $x$ axis: Operating with $\hat{P}_{y}$ on this component corresponds to swapping the particles located at the two other vertices of the triangle symmetrically placed above and below the $x$-axis; for bosons and fermions this will give the eigenvalue +1 and -1 respectively for this component. Since the Wigner state has to be an eigenfunction of $\hat{P}_{y}$, all other components of the wave function corresponding to tilted triangles must come in symmetric/antisymmetric pairs mirrored in the $x$-axis, so that the total wavefunction has the eigenvalue +1 (bosons) or -1 (fermions) for $\hat{P}_{y}$. The same analysis applies to $\hat{P}_{x}$ and we conclude that for the triangular Wigner state $\left(P_{x}, P_{y}\right)_{\mathrm{B} \text {, triangle }}=(+,+)$ for bosons and $\left(P_{x}, P_{y}\right)_{\mathrm{F} \text {,triangle }}=(-,-)$ for fermions. Since energy levels corresponding to wave functions of the same sym-
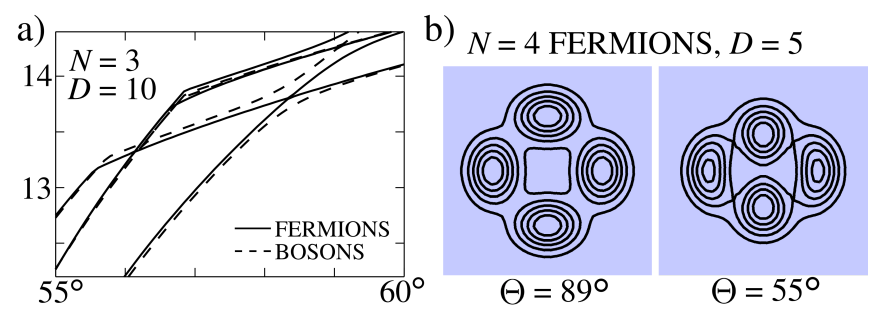

Figure 4: (a) The four lowest energy states for very strong interaction (axes as in Fig. 3). (b) Density contours for $N=4$ dipolar fermions.

metries cannot cross, the arguments above explain why the line-triangle transition for bosons corresponds to an avoided crossing, while it is sharp for fermions. It is perhaps surprising that quantum statistics plays an important role for the transitions between essentially classical Wigner states. This can, however, be understood by noting that the particles can overlap during the transitions meaning that quantum statistics matters.

It is illuminating to connect the states in Fig. 3 to the eigenstates of the angular momentum $\hat{L}_{z}$ along the $z$-axis for $\Theta=90^{\circ}$ : The lowest level in Fig. 3 naturally develop into to $L_{z}=0$ for $\Theta=90^{\circ}$, whereas the next two levels correspond to $L_{z} \pm 3$ and not $L_{z}= \pm 1$ as one would perhaps expect. This is because the strong repulsion favors states with large $|m|$ which are more spatially extended.

In Fig. 4 (a), we plot the four lowest energy states in the limit of very strong interaction $D=10$ (the interaction strength scales as $D^{2}$ ). Comparing with Fig. 3, we see that the difference between the bosons and fermions decreases with increasing coupling. This is as expected since the system approaches classical behavior in this limit. Reflecting this, the transition regions between different ground states where quantum statistics is important are smaller for $D=10$ than for $D=5$ : The size of the discussed anticrossing is $\Delta E=0.53$ for $D=5$ and $\Delta E=0.22$ for $D=10$. Also, the transition region has moved to larger angle $\Theta \simeq 59^{\circ}$ approaching the classical value $\Theta \simeq 62^{\circ}$ with increasing $D$.

In Fig. 4 (b), we plot the density of the ground state of $N=4$ fermionic dipoles for $D=5$. In this case, the dipoles localize in a rhombic geometry that depends on the tilt angle. To examine the case of higher particle numbers, we present in Fig. 5 the result of a classical minimization of the interaction and trap energy for $N=19$ dipoles. The ground state configurations were found using an iterative self-consistent procedure adjusting the particle positions to find the minimal energy. We see that in analogy with the $N=3,4$ cases considered above, the geometry of the lattice depends on the tilt angle. For $\Theta=90^{\circ}$, the particles localize in a hexagonal lattice 13. These classical calculations indicate that our main conclusions for quantum-mechanical few-body systems should be relevant also for larger systems.

The strong coupling effects considered here are reach- 


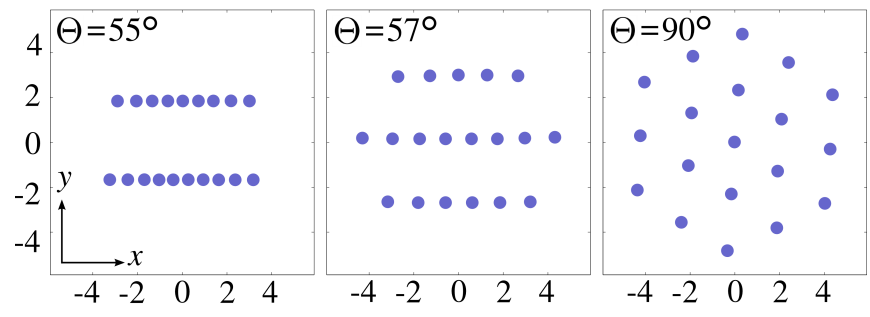

Figure 5: A classical calculation of the minimum energy configurations of $N=19$ dipoles in a $2 \mathrm{D}$ trap.

able using typical experimental numbers. The KRb molecule trapped by the JILA group [7] has an electric dipole moment of 0.57 Debye whereas the RbCs molecule studied by the Innsbruck group has an electric dipole moment of 1.25 Debye [17, 18. Trapping lengths of $l_{0}=1 \mu \mathrm{m}$ or $l_{0}=0.1 \mu \mathrm{m}$ yield coupling strengths between $0.6 \lesssim D^{2} \lesssim 50$. Also, there are alkali dimers with even larger dipole moments [6]. Using optical lattices, few-body systems with ultracold molecules may be realized, see e.g. the experiment in Ref. [4].

To summarize, we studied systems of strongly interacting bosonic and fermionic dipoles in a 2D harmonic trap, using both exact diagonalization and classical analysis. The dipolar interaction was shown to lead to a rich variety of Wigner states with nontrivial geometries which depend on the tilt angle of the dipoles with respect to the plane. Even though the Wigner states themselves can be well understood from a classical analysis, the transitions between different geometries as the tilt angle is changed depends crucially on the quantum statistics of the dipoles. We showed how the effects analyzed here are relevant for typical experimental parameters.

We thank G. Carlsson, J. Grönqvist, O. Karlström and F. Malet for helpful comments and suggestions. This work was financially supported by the Swedish Research Council and the Kungl. Fysiografiska Sällskapet in Lund.
[1] T. Lahaye et al., Nature (London) 448, 672 (2007)

[2] T. Koch et al., Nature Phys. 4, 218 (2008)

[3] M. Lu, S. H. Youn, and B. L. Lev, Phys. Rev. Lett. 104, 063001 (2010)

[4] J. G. Danzl et al., Nature Phys. 6, 265 (2010)

[5] J. Deiglmayr et al., New J. Phys. 11, 055034 (2009)

[6] M. Aymar and O. Dulieu, J. Chem. Phys. 122, 204302 (2005)

[7] C. Ospelkaus et al., Phys. Rev. Lett. 97, 120402 (2006); S. Ospelkaus et al., Nature Physics 4, 622 (2008); K.-K. Ni et al., Science 322, 231 (2008); S. Ospelkaus et al., Phys. Rev. Lett. 104, 030402 (2010)

[8] M. A. Baranov, Phys. Rep. 464, 71 (2008)

[9] T. Lahaye, C. Menotti, L. Santos, M. Lewenstein, and T. Pfau, Rep. Prog. Phys. 72, 126401 (2009)

[10] G. M. Bruun and E. Taylor, Phys. Rev. Lett. 101, 245301 (2008)

[11] K. Osterloh, N. Barberan, and M. Lewenstein, Phys. Rev. Lett. 99, 160403 (2007); G. E. Astrakharchik, J. Boronat, I. L. Kurbakov, and Y. E. Lozovik, Phys. Rev. Lett. 98, 060405 (2007); N. R. Cooper and G. V. Shlyapnikov, Phys. Rev. Lett. 103, 155302 (2009); B. M. Fregoso and E. Fradkin, Phys. Rev. Lett. 103, 205301 (2009); K. Sun, C. Wu, and S. Das Sarma, Phys. Rev. B 82, 075105 (2010)

[12] M. A. Baranov, H. Fehrmann, and M. Lewenstein, Phys. Rev. Lett. 100, 200402 (2008)

[13] H. P. Büchler et al., Phys. Rev. Lett. 98, 060404 (2007)

[14] E. P. Wigner, Phys. Rev. 46, 1002 (1934)

[15] F. Bolton and U. Rößler, Superlatt. Microstr. 13, 139 (1993); V. M. Bedanov and F. M. Peeters, Phys. Rev. B 49, 2667 (1994); S. M. Reimann and M. Manninen, Rev. Mod. Phys. 74, 1283 (2002); C. Yannouleas and U. Landman, Rep. Progr. Phys. 70, 2067 (2007); S. Kalliakos et al., Nature Physics 4, 467 (2008); F. Cavaliere, U. De Giovanni, M. Sassetti, and B. Kramer, New J. Phys. 11, 123004 (2009)

[16] S. Yi, H. Pu, Phys. Rev. A 73, 061602(R) (2006); S. Komineas, N. R. Cooper, Phys. Rev. A 75, 023623 (2007)

[17] K. Pilch et al., Phys. Rev. A 79, 042718 (2009)

[18] S. Kotochigova and E. Tiesinga, J. Chem. Phys. 123, 174304 (2005) 